Kazuya Minato $\cdot$ Ryosuke Takazawa $\cdot$ Kenji Ogura

\title{
Dependence of reaction kinetics and physical and mechanical properties on the reaction systems of acetylation II: physical and mechanical properties
}

\begin{abstract}
Acetylation of wood was carried out in acetic anhydride only, acetic anhydride/xylene 1:1 (v/v), and acetic anhydride/pyridine 4:1 (v/v) solutions. The antishrink efficiency (ASE), hygroscopic properties, vibrational properties, and bending strength were compared among the three reaction solutions. The ASE was a simple function of weight gain (WG); the equilibrium moisture content at a given WG differed among the reaction solutions. Based on this fact and the results of repeated water soaking and ovendrying tests, it was found that the bulking effect was a major factor, and that decreased hygroscopicity contributes only slightly to the dimensional stabilization by acetylation. The difference in equilibrium moisture content among reaction solutions appears more significant in block samples than wood meal, probably due to the fiber-to-fiber bonds in the former. The tendencies for change in the specific Young's modulus and the loss tangent differed among reaction solutions, whereas in the static bending test the difference was not marked.
\end{abstract}

\section{K. Minato $(\square)$}

Graduate School of Agriculture, Kyoto Prefectural University,

Sakyo-ku, Kyoto 606-8522, Japan

Tel. +81-75-703-5646; Fax +81-75-703-5646

e-mail: minato@kpu.ac.jp

R. Takazawa ${ }^{1}$

Faculty of Agriculture, Kyoto Prefectural University, Kyoto 606-8522, Japan

K. Ogura ${ }^{2}$

Graduate School of Agriculture, Kyoto Prefectural University, Kyoto 606-8522, Japan

\section{Present addresses}

${ }^{1}$ Wood Research Institute, Kyoto University, Uji 611-0011, Japan

${ }^{2}$ Sumitomo Forestry Co., Tsukuba Research Institute, Tsukuba 300-2646, Japan

Part of this report was represented at the 52nd Annual Meeting of the Japan Wood Research Society, Gifu, April 2002
Key words Acetylation - Dimensional stability $\cdot$ Moisture adsorption $\cdot$ Vibrational property $\cdot$ Mechanical strength

\section{Introduction}

In a previous paper, reaction parameters were compared among three reaction systems for acetylation of wood. Significant differences were observed in the rate constant, ultimate weight gain (WG), and activation energy depending on the reaction system, all explained in connection with the rate-determining step of acetylation. That is, in the acetic anhydride/xylene system, where the rate constant was smallest, access by the reagent to the reaction site precedes the true reaction, whereas in the acetic anhydridepyridine system, whose rate constant was greatest, it was suggested that the supply of reagent could not follow the reaction. The difference in the rate-determining step must influence the physical and mechanical properties of acetylated wood.

Generally, it is thought that the reaction of fibrous materials proceeds from the lumen and intercellular layer to the inner cell wall. Such a situation was observed in the crosslinking of cotton with formaldehyde..$^{1,2}$ Thus, during the slower uncatalyzed reaction the reaction expands uniformly to the whole cell wall. In contrast, during the rapid catalyzed reaction, the reaction was localized at the periphery of the cell wall and the vicinity of the cell lumen. The heterogeneous distribution of the reaction portion is also expected for acetylation and must result in differences in the dimensional stabilizing effect, moisture adsorption ability, mechanical properties, and so on. In previous studies, various physical and mechanical properties resulting from chemical treatment were often related to the degree of the reaction (e.g. WG) however, these properties have seldom been discussed in connection with the reaction system. In this study we intended to clarify the differences in the dimensional stabilizing efficiency, moisture adsorption ability, and mechanical properties for some typical acetylation systems. 


\section{Experiments}

Materials and acetylation

Sitka spruce (Picea sitchensis Carr.) was used as a 150 $355 \mu \mathrm{m}$ wood meal, $25 \mathrm{~mm}(\mathrm{~T}) \times 30 \mathrm{~mm}(\mathrm{R}) \times 5 \mathrm{~mm}(\mathrm{~L})$ cross sections, and $1.7 \mathrm{~mm}(\mathrm{~T}) \times 11 \mathrm{~mm}(\mathrm{R}) \times 120 \mathrm{~mm}(\mathrm{~L})$ strips for moisture adsorption tests, determination of dimensional stability, and evaluation of mechanical properties, respectively. Each sample was extracted with an alco$\mathrm{hol} / \mathrm{benzene}(\mathrm{v} / \mathrm{v}, 1: 2)$ mixture $(8 \mathrm{~h}$ for wood meal and $24 \mathrm{~h}$ for block and strip samples) and successively with alcohol ( $24 \mathrm{~h}$ for block and strip samples) by Soxhlet's extractor. The acetylation was carried out in acetic anhydride, an acetic anhydride/xylene mixed solution (v/v 1:1), and an acetic anhydride/pyridine mixed solution ( $\mathrm{v} / \mathrm{v}$ 4:1). Hereafter these solutions are referred to as the uncatalyzed, xylene, and pyridine systems, respectively. The details of the other reaction conditions are as previously described.

Tests of water soaking and drying

The cross-sectional samples were subjected to repeated measurements of dimensional change between watersoaked and oven-dried states. The two states were achieved by (1) aspiration until all bubbling ceased (about $4 \mathrm{~h}$ at room temperature) and (2) heating in an oven at $105^{\circ} \mathrm{C}$ for $2 \mathrm{~h}$. The dimensions were measured at two points for each tangential and radial direction and were expressed as the mean. The oven-dried length before treatment and the water-swollen length just after treatment are represented by $D_{0}$ and $W_{0}$, respectively, with the subscript indicating the number of repeats. Shrinking $(S)$ was calculated based on $W_{2}$ and $D_{3}$ as follows.

$S(\%)=\left(1-\frac{D_{3}}{W_{2}}\right) \times 100$

The antishrink efficiency (ASE) was estimated by the shrinking of untreated $\left(S_{\mathrm{u}}\right)$ and treated $\left(S_{\mathrm{t}}\right)$ specimens according to the following equation.

$\operatorname{ASE}(\%)=\left(1-\frac{S_{\mathrm{t}}}{S_{\mathrm{u}}}\right) \times 100$

Moisture adsorption test

For the untreated and acetylated wood meal, moisture adsorption isotherms were made for the nine kinds of saturated salt solutions at $20^{\circ} \mathrm{C}$ in a desiccator. The equilibrium moisture content was separately obtained from the specimen for mechanical tests at $20^{\circ} \mathrm{C}$ and $65 \%$ relative humidity $(\mathrm{RH})$.

Measurement of vibrational properties

The specific dynamic Young's modulus (E/ $\gamma$ : dynamic Young's modulus divided by the specific gravity) and loss tangent $(\tan \delta)$ were determined by a free-free flexural vibration method. ${ }^{3}$ The degree of change (percent) by the treatment was estimated on the basis of the value before treatment for the same specimen.

\section{Static bending test}

Specimens that completed the vibrational test were subjected to a static bending strength test. The test was carried out at $20^{\circ} \mathrm{C}$ and $65 \% \mathrm{RH}$, a span length of $100 \mathrm{~mm}$, and a crosshead speed of $5 \mathrm{~mm} / \mathrm{min}$. The modulus of rupture (MOR) and the modulus of elasticity (MOE) were obtained from the strain-stress curve.

\section{Results and discussion}

Dependence of dimensional change and ASE on the reaction system

Figure 1 shows an example of the dimensional change during repeated tests in the water-soaked and oven-dried states. The ordinate represents the real length of the specimens. For specimens treated by the uncatalyzed system (Fig. 1b), the length just after treatment $\left(W_{0}\right)$ was somewhat greater than the water-swollen length after drying $\left(W_{1}\right)$,
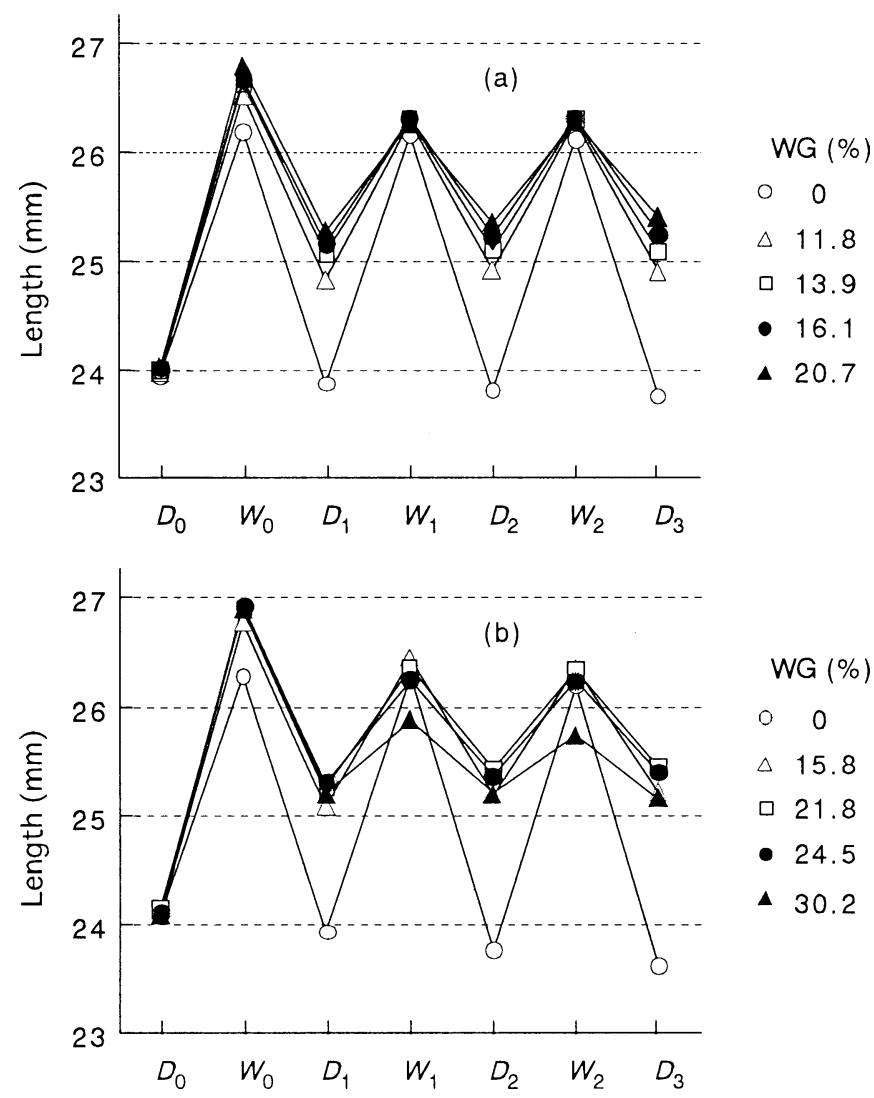

Fig. 1. Dimensional changes on repeated water soaking and oven drying for the uncatalyzed system (a) and the pyridine system (b). $W G$, weight gain 


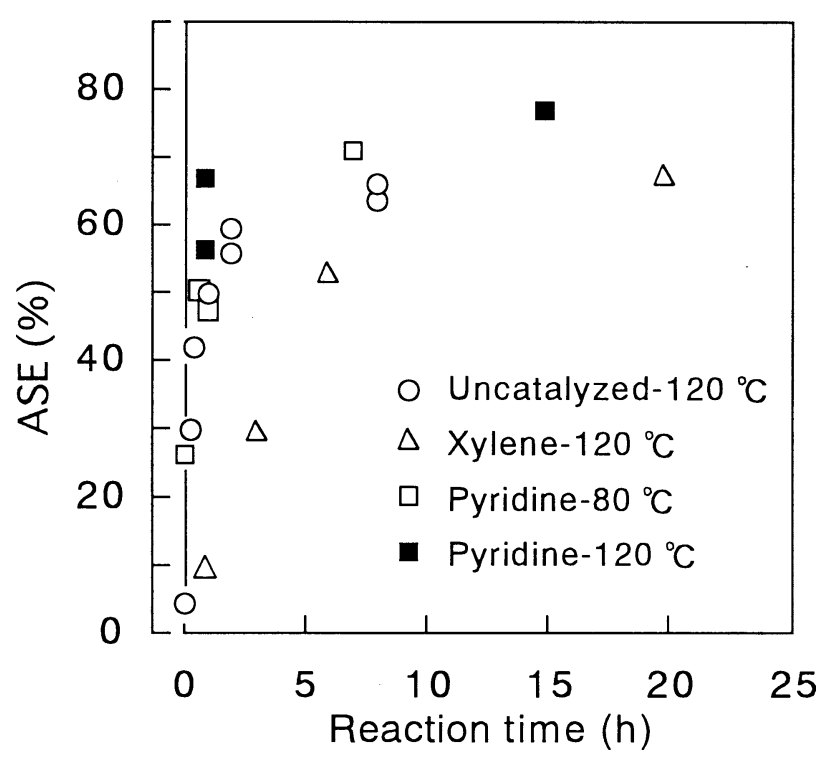

Fig. 2. Reaction profiles regarding the antishrink efficiency $(A S E)$ in the tangential drection

which suggests that the specimens swell more in the reaction solution than in water. At the $D_{1}$ stage, treated specimens were much longer than untreated specimens, whereas at $W_{1}$ the difference between the two was not significant. Therefore, the dimensional stabilizing effect of acetylation is virtually attributable to the bulking of the substituted acetyl groups. Even after repeated water soaking and drying, both dry and wet lengths of treated specimens remain unchanged. On the other hand, the dry lengths of untreated and lightly treated specimens decreased, probably owing to the elution of some wood component. The specimens treated in the xylene system behaved like those in the uncatalyzed system.

When the acetylation was carried out in a pyridine system (at $120^{\circ} \mathrm{C}$ for $14 \mathrm{~h}$ ), both water-swollen and oven-dried lengths decreased (Fig. 1b). In this case the darkening of the specimen suggests severe degradation.

The ASE estimated in the tangential direction is plotted against the reaction time in Fig. 2. The reaction rate at $120^{\circ} \mathrm{C}$ was greatest in the pyridine system, followed by the uncatalyzed and then the xylene system, which is the same order obtained from WG data for wood meal. A similar tendency was confirmed for the ASE in the radial direction.

Figure 3 shows the relation between WG and ASE in the tangential direction. The ASE attained with a definite WG value differed little among the reaction systems.

Dependence of moisture adsorption characteristics on the reaction system

Figure 4 shows an example of isotherms for the wood meal treated at various WG levels in the uncatalyzed system. The symbols and solid lines represent the observed values and calculated curves, respectively. A marked decrease of hygroscopicity was observed depending on the degree of

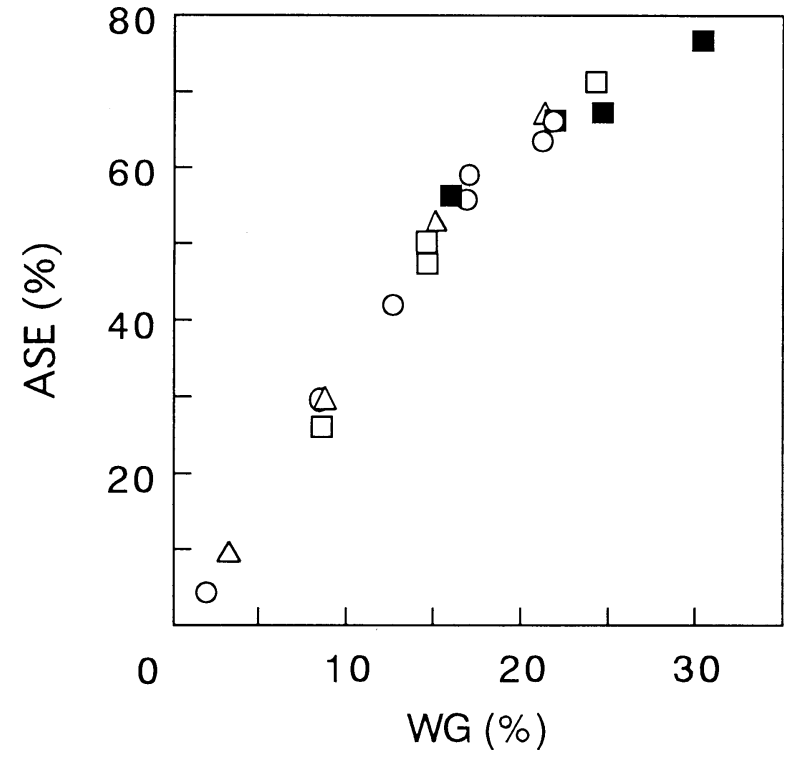

Fig. 3. Relation between WG and ASE in the tangential direction. Symbols are the same as in Fig. 2

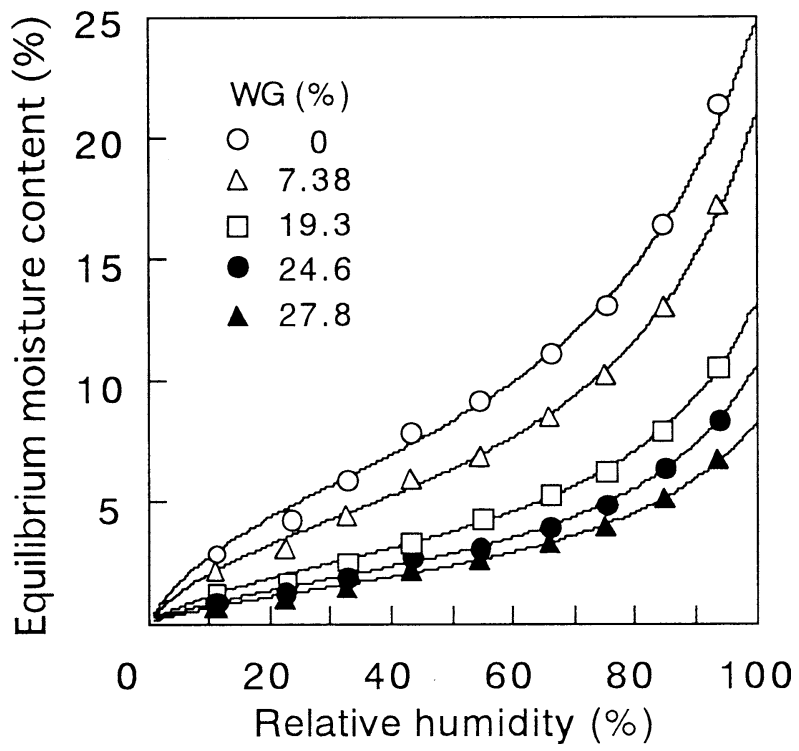

Fig. 4. Examples of isotherms drawn for wood meal treated in the uncatalyzed system

acetylation (Fig. 4). This does not exclude the possibility that the dimensional stabilizing effect of acetylation mainly originates from the bulking effect. This can be seen in Fig. 1, where the length in the water-swollen state did not differ considerably depending on the degree of reaction.

For a comparison of moisture adsorption ability among reaction systems, the observed equilibrium moisture content at $54.6 \% \mathrm{RH}$ (determined over a saturated solution of magnesium nitrate) was plotted against WG (Fig. 5). The equilibrium moisture content decreased with increasing WG, although there were no significant differences among reaction systems or reaction temperatures. 


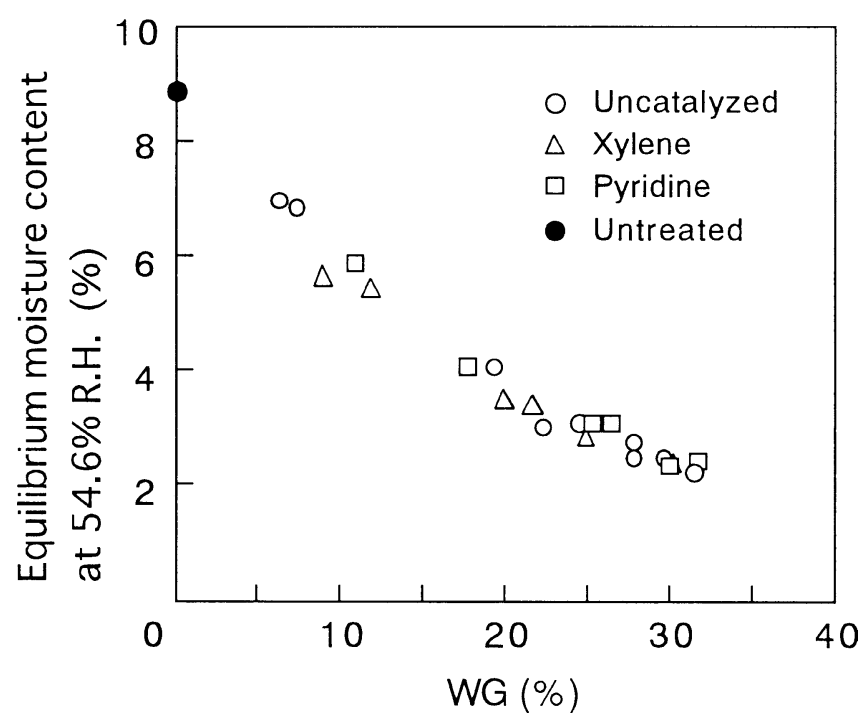

Fig. 5. Equilibrium moisture content determined at $54.6 \%$ relative humidity $(R H)$ for wood meal

Although the total moisture content did not differ among the reaction systems, the adsorbed water can be separated into two types, which form a monolayer and a multilayer; their share would differ among the reaction conditions, which in turn alter the degree of swelling and the state of the inner surface. Therefore, the isotherms were analyzed based on a theory proposed by Hailwood and Horrobin. ${ }^{4}$ With this theory, the total adsorbed water can be divided into hydrated water $\left(M_{\mathrm{h}}\right)$ and dissolved water $\left(M_{\mathrm{d}}\right)$, which correspond to the monolayer and multilayer adsorbed water, respectively. By conventional methods, a parabolic function was applied to the regression of observed values, and the constants $M_{\mathrm{h}}$ and $M_{\mathrm{s}}$ were calculated.

The curves in Fig. 4 were drawn based on the sum of $M_{\mathrm{h}}$ and $M_{\mathrm{s}}$. Although $M_{\mathrm{h}}$ and $M_{\mathrm{s}}$ can be calculated in any relative humidity, for convenience those at $54.6 \% \mathrm{RH}$ were plotted against WG (Fig. 6). When $M_{\mathrm{h}}$ and $M_{\mathrm{s}}$ were compared at a definite WG, $M_{\mathrm{h}}$ was slightly lower in the xylene system and $M_{\mathrm{s}}$ was higher in the pyridine system, probably due to the degree of swelling in the reaction system.

The equilibrium moisture contents determined for the wood blocks at $20^{\circ} \mathrm{C}$ and $65 \% \mathrm{RH}$ were also plotted against WG (Fig. 7). In this case the difference among reaction systems was marked, and the moisture content was lower in the xylene system than the other systems. It is unlikely that the WG was underestimated based on the elution of extractives in the xylene system, as the extractive content was $2 \%$ even if Soxhlet's extraction was incomplete. The reason for the differences among reaction systems is clear in the block sample and can be explained as follows. In the block sample each wood fiber remains tightly together, which might limit the extent of the reaction or accessibility of water molecules to the adsorption site to different degrees depending on the swelling of the wood in the reaction solutions. At any rate, the moisture adsorption ability of acetylated wood varied depending on the reaction system when the equilibrium moisture content was compared at a definite WG value.
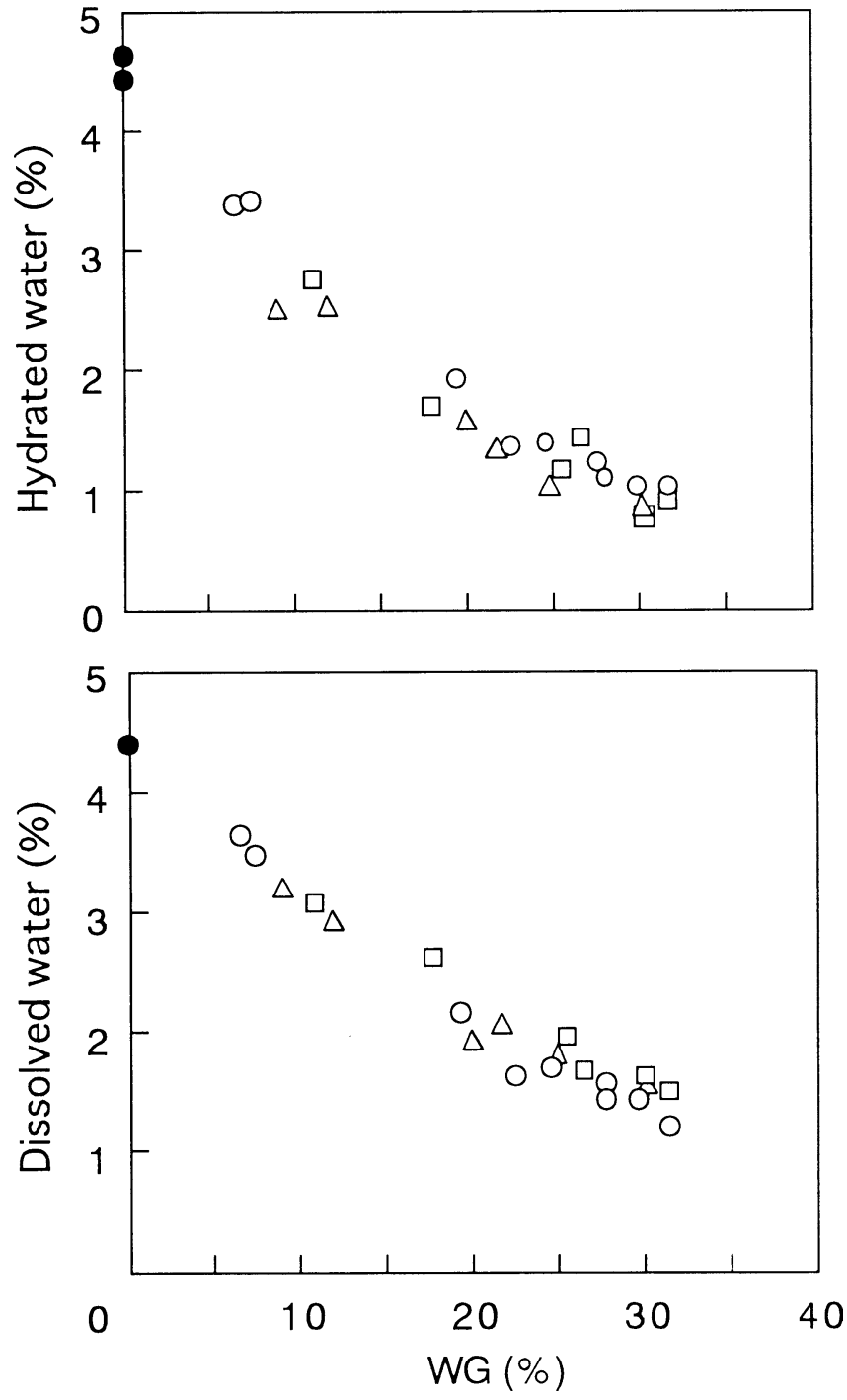

Fig. 6. Hydrated $\left(M_{\mathrm{h}}\right)$ and dissolved water $\left(M_{\mathrm{s}}\right)$ calculated at $54.6 \%$ relative humidity $(\mathrm{RH})$ for wood meal. Symbols are the same as in Fig. 5

The dependence of ASE and equilibrium moisture content on the reaction system may be related to the mechanism of dimensional stabilization. That is, the dimensional stability of acetylated wood mostly stems from the bulking of the acetyl groups taken into the cell wall. On the other hand, the decrease of hygroscopicity contributes only slightly to dimensional stabilization.

Dependence of mechanical properties on the reaction system

The specific dynamic Young's modulus $(E / \gamma)$ is plotted against WG in Fig. 8a. For all reaction systems, $E / \gamma$ decreased with increasing WG, mainly due to the increased $\gamma$. At a given WG, the order of lowering the $E / \gamma$ was pyridine system $>$ uncatalyzed system $>$ xylene system, which was consistent with the swelling of wood in the reaction solution. Especially in the pyridine system, it is likely that exces- 


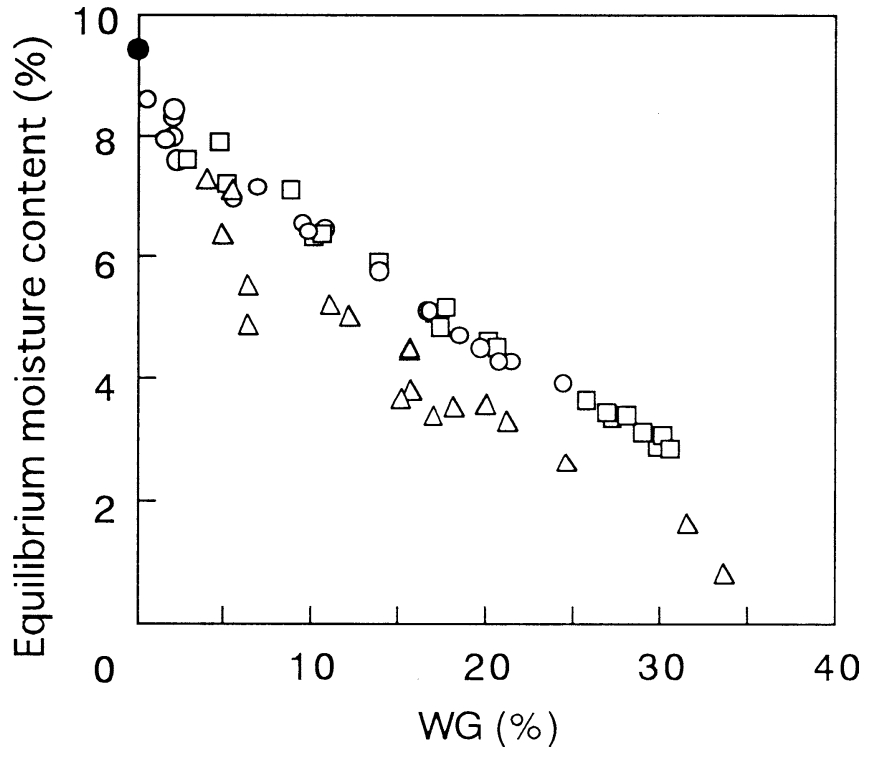

Fig. 7. Equilibrium moisture content determined at $20^{\circ} \mathrm{C}, 65 \% \mathrm{RH}$ for wood blocks. Symbols are the same as in Fig. 5
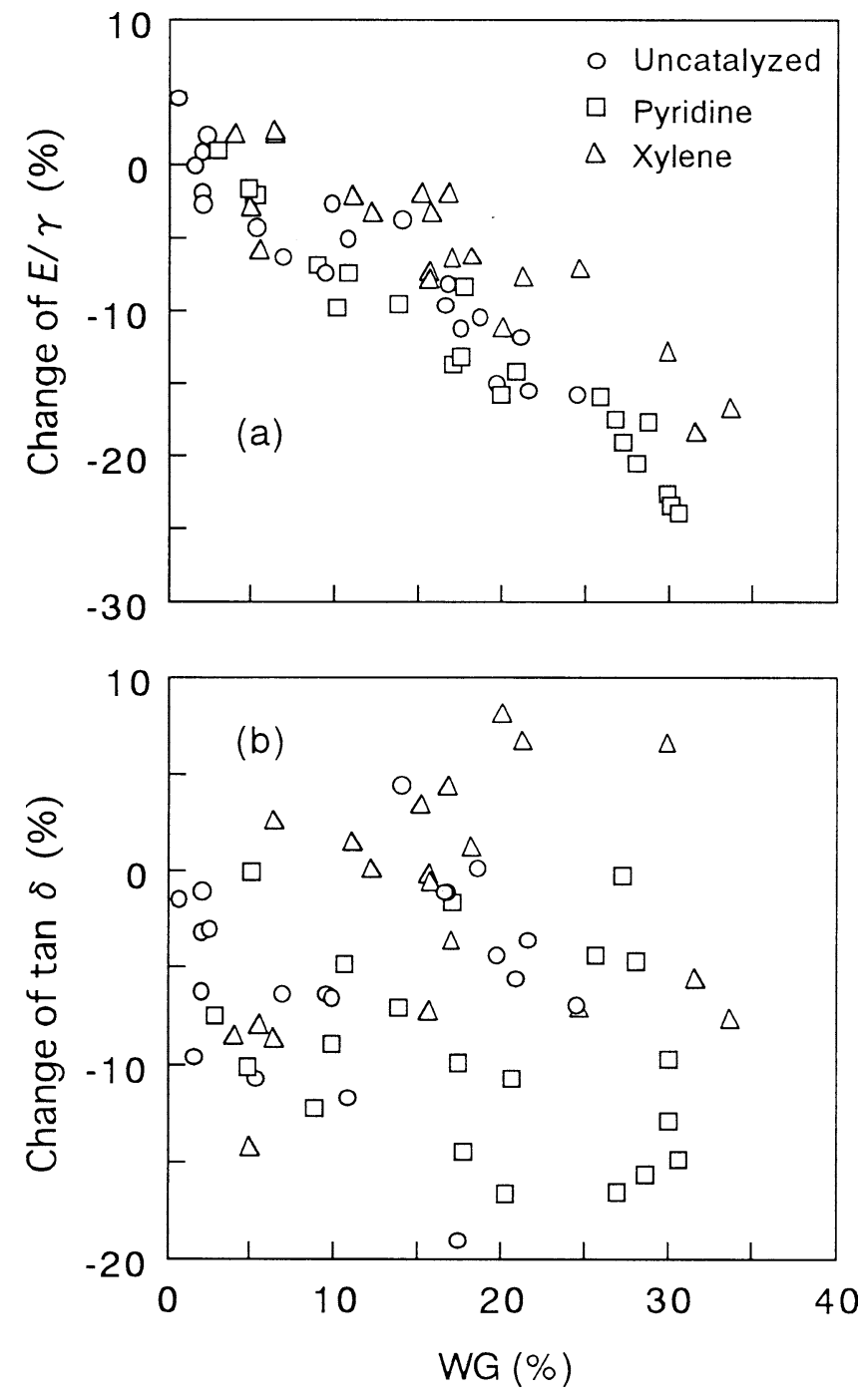

Fig. 8. Percent change of specific Young's modulus (a) and loss tangent (b) resulting from acetylation
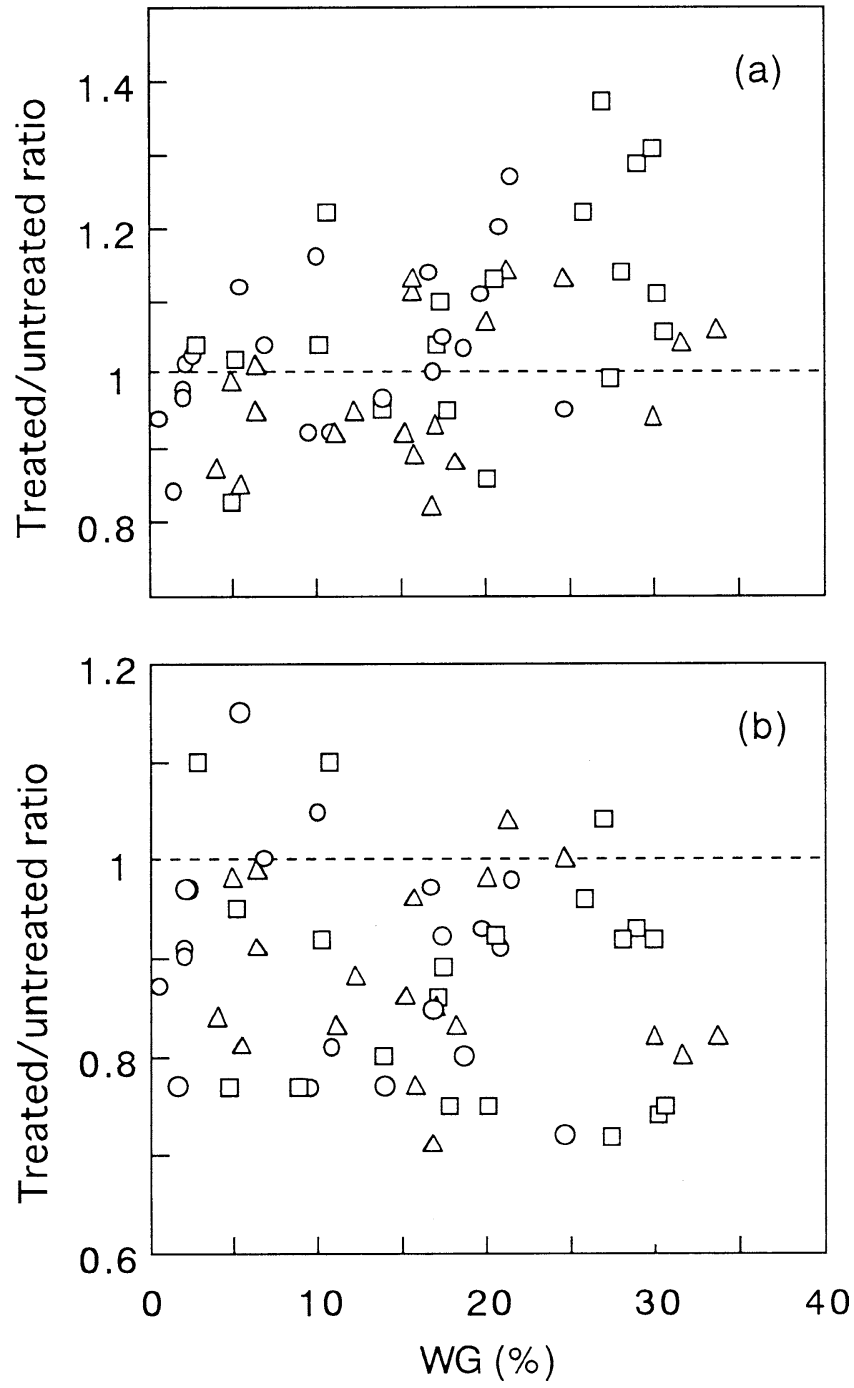

Fig. 9. Treated/untreated ratio of the modulus of rupture (a) and the modulus of elasticity (b) of the specimens acetylated in different reaction systems. Symbols are the same as in Fig. 8

sive swelling causes damage to the cell wall structure, which leads to decreases in Young's modulus and strength. On the other hand, in the xylene system the reaction solution might permeate the cell wall uniformly because of the slower reaction, which is not controlled by the transfer of reagent.

The change in $\tan \delta$ is plotted against WG in Fig. 8b. The $\tan \delta$ tends to decrease as a whole in the uncatalyzed and pyridine systems and seldom increases above the value before treatment. On the other hand, in the xylene system a slight increase was observed for many specimens, though the data were widely dispersed. The equilibrium moisture content was lower in the xylene system than the other systems, further confirming that the moisture content increases $\tan \delta$ above a given value. Therefore, this cannot explain the result found in the xylene system. More precise data are required to discuss the change in $\tan \delta$ with acetylation.

Figure 9 shows the results of the static bending test. The MOR tends to increase with increasing WG, and at a given level it is slightly lower in the xylene system than in the 
other systems. A significant difference was not observed in the MOE among the reaction systems.

\section{Conclusions}

Acetylation was carried out in uncatalyzed, xylene, and pyridine systems; physical and mechanical properties were compared among the systems. The ASE level was determined only by WG and was independent of the reaction system, whereas the equilibrium moisture content differed among the reaction systems, probably due to the difference in swelling in the reaction mixture. This is attributable to the fact that the dimensional stability of acetylated wood mostly stems from the bulking of substituted acetyl groups, whereas decreased hygroscopicity is not a major factor in the dimensional stabilization by acetylation. The differences in the reaction systems had a complicated influence on the mechanical properties, and additional tests are necessary for further discussion.

\section{References}

1. Joarder GK, Brannan AF, Rowland SP, Guthrie JD (1969) An uncatalyzed, vapor-phase crosslinking reaction of cotton cellulose with formaldehyde. Textile Res J 39:49-54

2. Cannizzaro AM, Goynes WR, Rollins ML, Keating EJ (1970) Microscopical studies of cottons reacted in vapor phase. Textile Res J 40:1087-1095

3. Hearmon RFS (1966) Theory of the vibrational testing of wood. Forest Prod J 16:29-40

4. Hailwood AJ, Horrobin S (1946) Absorption of water by polymers: analysis in terms of a simple model. Trans Farad Soc 42B:84-92 